\title{
Melatonin Increases the Anticancer Potential of Doxorubicin in Caco-2 Colorectal Cancer Cells
}

\author{
Mahdieh Farhoudi Sefidan Jadid \\ Islamic Azad University Tabriz Branch \\ Elnaz Aghaei \\ Islamic Azad University Arsanjan
}

\section{Elham Taheri}

Tabriz University of Medical Sciences

\section{Nasrin Seyyedsani}

Islamic Azad University Tabriz Branch

\section{Reza Chavoshi}

Islamic Azad University Tabriz Branch

\section{Samane Abbasi}

University of Guilan

\section{Afshin Khorrami}

Yazd University

\section{Saba Hajazimian}

Tabriz University of Medical Sciences

\section{Sina Taefehshokr}

Tabriz University of Medical Sciences

Alireza Isazadeh ( $\boldsymbol{Q}$ isazadeh.alireza73@gmail.com )

tabriz university of medical sciences

Dariush Shanehbandi

Tabriz University of Medical Sciences

\section{Original article}

Keywords: Colorectal Cancer, Combination therapy, Doxorubicin, Melatonin

Posted Date: October 12th, 2020

DOl: https://doi.org/10.21203/rs.3.rs-89371/v1

License: (a) This work is licensed under a Creative Commons Attribution 4.0 International License. Read Full License 


\section{Abstract}

Colorectal cancer (CC) is an important human malignancy with high cancer related death worldwide. The chemotherapy using doxorubicin hydrochloride is one of the most common cancer therapeutic methods. However, drug resistance lowers the treatment efficacy in CC patients. The combination therapies seem to be more promising by taking the advantage of synergistic effects. The present study aimed to evaluate a new strategy to enhance the anticancer activity of doxorubicin in Caco-2 CC cell line by co-administration of melatonin. The effects of doxorubicin, melatonin, and their combinations (Dox-Mel) were investigated on the proliferation and viability, morphological alterations, and tumor spheroid formation. Flow cytometry was employed to compare the apoptotic situation of the cells in study groups. Changes in metastatic potential of the cells were assessed by wound healing assay and trans-well migration assays. Moreover, expression of BAX, SMAC, BCL-2, SURVIVIN, MMP-2, and MMP-9 genes were evaluated by quantitative real time PCR (qRT-PCR) and western blotting. Our study showed that doxorubicin, melatonin, and Dox-Mel significantly decreased the proliferation and viability, tumor spheroid formation, invasion and migration. Furthermore, the changes were in a concentration and time dependent manner. There was an increase in apoptosis rate in the treatment groups. Expression of genes involved in apoptosis and cell motility were altered significantly. It was observed that anticancer activity of Dox-Mel combination was significantly more than doxorubicin and melatonin treatments alone. We showed an enhanced apoptotic and anticancer activity of doxorubicin and melatonin combination chemotherapy on CC cell line than doxorubicin or melatonin treatments alone. This combination could promote the treatment efficiency and alleviate the un-intended side effects by lowering the dose of doxorubicin prescription.

\section{Introduction}

Colorectal cancer (CC) is a highly prevalent malignancy and causative of cancer related death in the world. The incidence of $\mathrm{CC}$ is much lower in the developing countries of Africa and Asia compared with the United States and developed European countries (Haggar and Boushey, 2009). Nowadays, surgery and chemotherapy are the most common methods for treatment of CC. However, due to development of drug-resistant CC cells the efficacy of chemotherapy is limited (Wang et al., 2017). Therefore, application of combinational methods seems to be more effective and result in prolonged survival of CC patients (Soheilyfar et al., 2018; Maroufi et al., 2020).

Doxorubicin is an anthracycline antibiotic, commonly used as a chemotherapeutic agent in treatment of patients with CC. This chemotherapy agent inhibits cancer cells through induction of apoptosis and inhibition of cell division. However, resistance to doxorubicin is frequently evident in several drug-resistant cancer cells (Nakanishi and Ross, 2012). The upregulation of efflux transporters decreases intracellular levels of doxorubicin through drug excretion. This leads to reduction of therapeutic efficacy and apoptosis rate in drug-resistant cancer cells. Accordingly, anticancer activity of doxorubicin decreases in cancer cells with inhibited apoptosis. Various molecular processes are involved in dysregulation of apoptotic pathways including: activation of anti-apoptotic factors (Bcl-2, Bfl1/A1, etc.), inactivation of pro-apoptotic factors (Smac, Bax, etc.), and reinforcement of survival signals (Survivin, NF-KB, etc.). 
Therefore, therapeutic efficacy of doxorubicin on cancer cells were decreased through overexpression of efflux transporters and inhibition of apoptosis (Hu et al., 206). Combination of doxorubicin with different compounds and molecules to overcome the drug resistance has been found to be promising in various cancer cells (Menéndez et al., 2019; Fic et al., 2007).

Melatonin is a natural hormone secreted by the pineal gland as well as testes, retina, ovary, skin, and intestine (Chen et al., 2016). Previous studies reported that, melatonin plays an important role in regulation of several biological pathways such as secretion and modulation of hormones, reproduction, and circadian rhythms (Kim et al., 2012; Wang et al., 2012; Maroufi et al., 2020). In addition, melatonin has shown an appropriate anticancer activity without side effects on several cancer cells (Wang et al., 2012; Maroufi et al., 2020). Melatonin activities are mediated by receptor-dependent or -independent pathways (Maroufi et al., 2020; Witt-Enderby et al., 2006). The most important melatonin receptors are MT1 and MT2, which inhibit several signaling pathways involved in cancer progression including phosphoinositide 3-kinases (PI3K), mitogen activated protein kinase (MAPK) pathways and stimulate apoptotic pathways in several cancer cells (Kong et al., 2008; Mao et al., 2010). Moreover, the anticancer activity of melatonin has been largely described in hormone-dependent tumors, when administered alone or in combination with therapeutic agents (Menéndez et al., 2018; Najafi et al., 2019).

So far, several studies have been conducted to assess the anticancer effects of various natural compounds in combination with doxorubicin against cancer cells. However, this is the first study on the anticancer effects of doxorubicin-melatonin combination and underlying mechanisms in CC cell line which can help making decisions for clinical applications in the future.

\section{Materials And Methods}

\section{Cancer cell culture}

The CC cell line Caco-2 was obtained from Immunology Research Center, Tabriz University of Medical Sciences. The cell culture was performed using Roswell Park Memorial Institute (RPMI)-1640 medium containing $10 \%$ fetal bovine serum (FBS) and appropriate concentrations of penicillin (5,000 units/mL)and streptomycin $(5,000 \mathrm{mg} / \mathrm{mL})$. The cells were then incubated in standard conditions $\left(5 \% \mathrm{CO}_{2}, 95 \%\right.$ humidity and $37^{\circ} \mathrm{C}$ ).

\section{Cancer cell viability assay}

The Caco- 2 cancer cells were seeded in 96 well culture plates $\left(1.5 \times 10^{4}\right.$ cells/well) in $200 \mu \mathrm{L}$ culture medium, and then incubated for 24 hours. Next, cancer cells were treated with different concentrations of doxorubicin $(1,2,4$, and $8 \mu \mathrm{M})$, melatonin $(1,2,4$, and $8 \mathrm{mM})$, and Dox-Mel $(1,2,4$, and $8 \mu \mathrm{M}-\mathrm{mM})$, and incubated for $24-72$ hours. Then, $5 \mathrm{mg} / \mathrm{mL}$ of MTT reagent incomplete medium was substituted with previous medium and incubated for 4 hours. Then, culture medium removed, and $50 \mu$ dimethyl sulfoxide was added, and incubated for 30 minutes. The optical densities (OD) were measured at $570 \mathrm{~nm}$ 
wavelength. Finally, viability of cancer cells was measured using this formula: [(treated sample OD / untreated sample OD) $\times 100]$.

\section{Assessment of the morphological alterations}

The Caco- 2 cancer cells were seeded in a 6 well culture plates $\left(1.5 \times 10^{5}\right.$ cells/well $)$ in $2000 \mu \mathrm{L}$ culture medium. After 24 hours of incubation, the cells were then treated with doxorubicin $(1 \mu \mathrm{M})$, melatonin (2 mM), and Dox-Mel (0.8 $\mu \mathrm{M}-\mathrm{mM})$, and then incubated for 24-72 hours. Finally, morphological alterations of cancer cells were monitored by an inverted phase contrast microscope.

\section{Spheroid formation assay}

The Caco-2cells were seeded in a6well culture plates $\left(1.5 \times 10^{5}\right.$ cells/well) in $2000 \mu \mathrm{L}$ culture mediumcontaining10 $\mu \mathrm{M}$ basic fibroblast growth factor (bFGF) and $10 \mu \mathrm{M}$ epidermal growth factor (EGF), and then incubated for 24 hours. Subsequently, the cancer cells were treated with doxorubicin $(1 \mu \mathrm{M})$, melatonin $(2 \mathrm{mM})$, and Dox-Mel $(0.8 \mu \mathrm{M}-\mathrm{mM})$, and maintained in an incubator under standard conditions. The culture medium was substituted every 72 hours. Finally, formation of tumor sphere-like cells was monitored by an inverted phase contrast microscope, after 7-12 days

\section{Wound healing assay}

The Caco- 2 cancer cells were seeded in a 6 well culture plates $\left(1.5 \times 10^{5}\right.$ cells/well $)$ in $2000 \mu \mathrm{L}$ culture medium, and then incubated. After reaching to more than $90 \%$ confluence, the cancer cell monolayers in each well were scratched with a sterile pipette tip. Then, cancer cells were treated with doxorubicin $(1 \mu \mathrm{M})$, melatonin (2 mM), and Dox-Mel $(0.8 \mu \mathrm{M}-\mathrm{mM})$, and incubated for 0-20 hours. Finally, migration of cancer cells was monitored by an inverted phase contrast microscope.

\section{Trans-well migration assay}

The Caco-2 cancer cells were seeded in the upper trans-well chamber in serum free culture medium and treated by doxorubicin $(1 \mu \mathrm{M})$, melatonin $(2 \mathrm{mM})$, and Dox-Mel $(0.8 \mu \mathrm{M}-\mathrm{mM})$ for $10-20$ hours. In parallel, supplemented culture medium was added to the bottom trans-well chamber, and incubated for 20 hours. Then, non-migrated cancer cells in the upper chamber were removed, and the migrated cancer cells in the bottom chamber were fixed by $4 \%$ paraformaldehyde and stained by $0.1 \%$ crystal violet. Finally, migration of cancer cells in 3 random fields was monitored by an inverted phase contrast microscope.

\section{Apoptosis quantification by Annexin V-FITC/PI assay}

The Caco-2cells were seeded in a6 well culture plates $\left(1.5 \times 10^{5}\right.$ cells/well $)$ in $2000 \mu \mathrm{L}$ culture medium. After 24 hours of incubation, the cells were treated with doxorubicin $(1 \mu \mathrm{M})$, melatonin $(2 \mathrm{mM})$, and DoxMel $(0.8 \mu \mathrm{M}-\mathrm{mM})$, and maintained for 48 hours. The cancer cells were detached, washed, and resuspended in binding buffer containing Annexin V-FITC $(5 \mathrm{~mL})$ and $\mathrm{PI}(10 \mathrm{~mL})$, and incubated at room temperature in dark for 15 minutes. Finally, apoptosis rate in cancer cells was measured by a flow cytometry instrument (Becton Dickinson Bioscience). 


\section{Gene expression assay by real time PCR}

The Caco- 2 cancer cells were seeded in a 6 well culture plates $\left(1.5 \times 10^{5}\right.$ cells/well $)$ in $2000 \mu \mathrm{L}$ culture medium, and then incubated overnight. Then, the cancer cells were treated with doxorubicin $(1 \mu \mathrm{M})$, melatonin $(2 \mathrm{mM})$, and Dox-Mel $(0.8 \mu \mathrm{M}-\mathrm{mM})$, and incubated for 48 hours. The cancer cells were detached, washed, and re-suspended in TRIzol reagent, and total RNA extraction was performed according to the manufacturer's instructions. Then, random hexamer primer was used for CDNA synthesis. The mRNA expression levels of BAX, BCL-2, SMAC, SURVIVIN, MMP-2, and MMP-9 genes were evaluated using quantitative Real Time PCR (qRT-PCR). The used primers sequences were presented in Table 1. The Real Time PCR was performed in $20 \mu$ total volume comprising: $1 \mu \mathrm{l}$ CDNA, $0.5 \mu \mathrm{l}$ forward and reverse primers $(5 \mu \mathrm{M})$, and $10 \mu$ of PCR pre-Mix (Takara, RR820L). The cycling program included: 1 cycle of 1 minutes at $94^{\circ} \mathrm{C}$ ) followed by 45 cycles of denaturation at $94^{\circ} \mathrm{C}$ for 10 seconds, annealing at $59{ }^{\circ} \mathrm{C}$ for 30 seconds, and extension at $72{ }^{\circ} \mathrm{C}$ for 20 seconds. The ACTB gene ( $\beta$-actin) was considered as endogenous control, and calculations were conducted by $2^{-\triangle \Delta C t}$ (Livak) formula.

Table 1

Sequences and characteristics of primers used for detection of genes mRNA expression.

\begin{tabular}{|c|c|c|c|}
\hline Genes & Primer sequence & $\mathrm{T}_{\mathrm{m}}$ & Products size \\
\hline \multirow[t]{2}{*}{$B A X$} & F-CCCGAGAGGTCTTTTTCCGAG & $60^{\circ} \mathrm{C}$ & \multirow[t]{2}{*}{$155 \mathrm{bp}$} \\
\hline & R-CCAGCCCATGATGGTTCTGAT & $60^{\circ} \mathrm{C}$ & \\
\hline \multirow[t]{2}{*}{$B C L-2$} & F-GATGGGATCGTTGCCTTATG & $56^{\circ} \mathrm{C}$ & \multirow[t]{2}{*}{$223 \mathrm{bp}$} \\
\hline & R-GCGGAACACTTGATTCTGG & $56^{\circ} \mathrm{C}$ & \\
\hline \multirow[t]{2}{*}{$S M A C$} & F-CAGAGGAGGAAGATGAAGTGTG & $55^{\circ} \mathrm{C}$ & \multirow[t]{2}{*}{$196 \mathrm{bp}$} \\
\hline & R-GCGGTTATAGAGGCCTGATCTG & $56^{\circ} \mathrm{C}$ & \\
\hline \multirow[t]{2}{*}{ SURVIVIN } & F-СССТTTCTCAAGGACCACCG & $52^{\circ} \mathrm{C}$ & \multirow[t]{2}{*}{172 bp } \\
\hline & R-GTTCCTCTATGGGGTCGTCA & $51^{\circ} \mathrm{C}$ & \\
\hline \multirow[t]{2}{*}{$M M P-2$} & F-CACATAGTGATGGTTCCСCTGTT & $60^{\circ} \mathrm{C}$ & \multirow[t]{2}{*}{$177 \mathrm{bp}$} \\
\hline & R-CGGCCACTCAGTAGGTGTCTTT & $62^{\circ} \mathrm{C}$ & \\
\hline \multirow[t]{2}{*}{$M M P-9$} & F-ATTTCTGCCAGGACCGCTTCTAC & $62^{\circ} \mathrm{C}$ & \multirow[t]{2}{*}{$187 \mathrm{bp}$} \\
\hline & R-ATCCGGCAAACTGGCTCCTTC & $63^{\circ} \mathrm{C}$ & \\
\hline \multirow[t]{2}{*}{ ACTIN } & F-AGAGCTACGAGCTGCCTGAC & $61^{\circ} \mathrm{C}$ & \multirow[t]{2}{*}{$186 \mathrm{bp}$} \\
\hline & R-AGCACTGTGTTGGCGTACAG & $59^{\circ} \mathrm{C}$ & \\
\hline
\end{tabular}

\section{Gene expression assay by western blotting}


The Caco- 2 cancer cells were seeded in a 6 well culture plates $\left(1.5 \times 10^{5}\right.$ cells/well $)$ in $2000 \mu \mathrm{L}$ culture medium, and then incubated for 24 hours. Then, the cancer cells were treated with doxorubicin $(1 \mu \mathrm{M})$, melatonin $(2 \mathrm{mM})$, and Dox-Mel $(0.8 \mu \mathrm{M}-\mathrm{mM})$, and incubated for 48 hours. The cancer cells were detached, washed, and lysed inice-cold RIPA lysis buffer containing protease inhibitor for 30 minutes. The cell lysates centrifuged and supernatant collected. The protein expression levels of Bax, Bcl-2, Smac, Survivin, MMP-2, and MMP-9 were evaluated using western blot analysis. Approximately $100 \mu \mathrm{g}$ of cells protein) were separated by sodium dodecyl sulfate-polyacrylamide gel electrophoresis (SDS-PAGE), and then transferred on to polyvinylidene difluoride (PVDF) membrane. The membrane was blocked using non-fat dry milk (5\%) and then incubated with primary antibodies at $4{ }^{\circ} \mathrm{C}$ for 24 hours. The horseradish peroxidase (HRP) conjugated secondary antibody was used as secondary antibody at room temperature for 60 minutes. The protein detection was performed using enhanced chemiluminescent (ECL) detection system.

\section{Statistical analysis}

The all performed experiments were repeated for three times, and obtained data were presented as mean \pm standard deviation (SD). The Student's ttest, Tukey (posthoc), and oneway analysis of variance (ANOVA) were used to statistical analysis using the Graph Pad Prism software. The $p$-value $<0.05$ was considered as significant.

\section{Results}

\section{Cancer cell viability}

According to the results, both doxorubicin and melatonin demonstrated a time and concentration dependent anticancer activity. The viability of the cancer cells was significantly decreased in the high concentrations of doxorubicin and melatonin. In addition, the anticancer activity of Dox-Mel was significantly more than doxorubicin and melatonin. The half maximal inhibitory concentration $\left(\mathrm{IC}_{50}\right)$ of doxorubicin, melatonin, and Dox-Mel on CC cells after 48 hours were: $1 \mu \mathrm{M}, 2 \mathrm{mM}$, and $0.8 \mu \mathrm{M}-\mathrm{mM}$, respectively (Fig. 1).

\section{Morphological alterations}

The cancer cells treated with doxorubicin, melatonin, and Dox-Mel showed several morphological alterations including shrinkage, decreased cell size, fragmented nuclei, and membrane damages, which can result in programmed cell death. The cells treated with Dox-Mel revealed significantly more morphological alterations than the cells with doxorubicin and melatonin monotherapy (Fig. 2).

\section{Tumor spheroid}

The treatment of cancer cells with doxorubicin, melatonin, and Dox-Mell showed significant decrease in the number and size of the spheroids compared to the untreated cancer cells. The size and number of 
tumor spheroids in the treated cancer cells with Dox-Mel were significantly less than in Caco-2 cells treated with doxorubicin and melatonin alone (Fig. 3).

\section{Cancer cell migration and invasion}

The trans-well assay showed that the migration and invasion of cancer cells treated with doxorubicin, melatonin, and Dox-Mel was significantly decreased in a time dependent manner (Fig. 4A). The wound healing showed a significant decrease in the migration and invasion of cancer cells treated with doxorubicin, melatonin, and Dox-Mel in a time dependent manner (Fig. 5A). However, inhibition of migration and invasion in the cancer cells treated with Dox-Mel was significantly more than doxorubicin and melatonin groups (Fig. 4B and Fig. 5B).

\section{Cancer cell apoptosis}

The Annexin V-FITC/PI assay showed that early and late apoptosis rate were significantly increased in cancer cells treated with doxorubicin, melatonin and Dox-Mel compared to untreated cells. Total apoptosis rate in the cancer cells treated with doxorubicin and melatonin were $34 \%$ and $29 \%$, respectively; whereas the total apoptosis rate was $42 \%$ in cancer cells treated with Dox-Mel combination (Fig. 6).

\section{Transcript quantification of migration and invasion related genes}

The mRNA expression levels of MMP-2 and MMP-9 genes were significantly decreased in treated Caco-2 cells with doxorubicin, melatonin, and Dox-Mel. However, regulation of migration and invasion related genes expression in the treated cancer cells with Dox-Mel was significantly more profound than cells treated with doxorubicin and melatonin (Fig. 7A, B).

\section{Expression analysis of migration and invasion related genes in protein level}

The migration and invasion related MMP-2 and MMP-9 protein levels significantly decreased in Caco-2 cells treated with doxorubicin, melatonin, and Dox-Mel. However, regulation of migration and invasion related proteins levels in the treated cancer cells with Dox-Mel was significantly more profound than treated cancer cells with doxorubicin and melatonin (Fig. 8).

\section{Transcript quantification of apoptosis related genes}

mRNA expression of the apoptosis related genes including BCL-2 and SURVIVIN significantly decreased in treated cancer cells with doxorubicin, melatonin, and Dox-Mel (Fig. 7C, D). Moreover, apoptosis related $B A X$ and SMAC genes mRNA expression significantly increased treated cancer cells with doxorubicin, melatonin, and Dox-Mel (Fig. 7E, F). However, regulation of apoptosis related genes expression in the 
treated cancer cells with Dox-Mel was significantly more profound than treated cancer cells with doxorubicin and melatonin.

\section{Expression analysis of apoptosis related genes in protein level}

The apoptosis related Bcl-2 and Survivin proteins levels significantly decreased in treated cancer cells with doxorubicin, melatonin, and Dox-Mel. Moreover, apoptosis related Bax and Smac proteins levels significantly increased in treated cancer cells with doxorubicin, melatonin, and Dox-Mel. However, regulation of apoptosis related proteins levels in the treated cancer cells with Dox-Mel was significantly more profound than treated cancer cells with doxorubicin and melatonin (Fig. 9).

\section{Discussion}

Resistance to chemotherapeutic drugs is one of the most important challenges in treatment of patients with cancer. Several molecular mechanisms are involved in progress of resistance to chemotherapeutic agents in patients with CC including drug efflux, drug inactivation, reduced prodrug activation, drug targets alteration, growth factors alterations, extracellular matrix, microRNAs and cytokines involvement, cell death and survival dysregulation, and hypoxia (Vahedian et al., 2020; Firouzi Amoodizaj et al., 2020). Recently, various natural compounds and their derivatives are being used to overcome the resistance to chemotherapeutic drugs through regulation of various mechanisms (Hajazimian et al., 2020; Mahdavi et al., 2019).

Doxorubicin is a widely used cancer drug for the treatment of a variety of human malignancies (Xu et al., 2018). However, overexpression of drug efflux transporters including multi drug resistance-associated protein 1 (MRP1), breast cancer resistance protein (BCRP), and P-glycoprotein (P-gp) has been reported to play important roles in mediating drug resistance in CC cells (Xu et al., 2018). Upregulation of these transporters results in decreased intracellular accumulation of anticancer drugs (Hu et al., 2016; Xu et al., 2018). Moreover, inhibition of apoptosis is a frequent event occurred in cancer cells resistant to doxorubicin. this happens through down-regulation of pro-apoptotic genes and/or up-regulation of antiapoptotic genes (Xu et al., 2018). Several studies demonstrated that, anticancer effects of doxorubicin increases in combination with other natural compounds (Xu et al., 2018; Hosseinzadeh et al., 2011). Previous studies have also reported that melatonin has an appropriate anticancer activity through regulation of several pathways, such as p53, MAPK, AMPK, and NF-KB as well as induction of apoptotic pathways (Kong et al., 2008; Mao et al., 2012). Therefore, it is important to evaluate whether melatonin enhances the anticancer effects of doxorubicin on CC cells.

In the present study, we assessed the anticancer effects of doxorubicin, melatonin, and Dox-Mel on CC cells and underlying mechanisms of action. We demonstrated that, melatonin increases the anticancer effects of doxorubicin against CC cells through regulation of several mechanisms involved in cell viability. 
We evaluated the morphological alterations and tumor spheroid formation in CC cells treated with doxorubicin, melatonin, and Dox-Mel in comparison with untreated CC cells. According to the results, various morphological alterations such as cell shrinkage, chromatin condensation, and apoptotic body formation as indicators of apoptosis induction were increased in CC cells treated with doxorubicin, melatonin, and Dox-Mel. However, morphological alterations of CC cells treated with Dox-Mel significantly more increased, as compared to doxorubicin and melatonin monotherapy. Moreover, tumor spheroid formation was significantly decreased in CC cells treated with Dox-Mel, as compared with doxorubicin and melatonin monotherapy.

The high lethality in patients with CC is due to metastasis and tumor growth at distant tissues (Bakhshaiesh et al., 2015). In this study, wound healing and trans-well migration assays demonstrated that invasion and migration of CC cells treated with Dox-Mel decreased more significantly, as compared doxorubicin or melatonin monotherapy. Moreover, we evaluated them RNA and protein levels of MMP-2 and MMP-9, which is related to CC cells metastasis and invasion. Our results showed that doxorubicin, melatonin, and Dox-Mel significantly inhibited the mRNA and protein levels of MMP-2 and MMP-9 in CC cells. However, the mRNA and protein levels of MMP-2 and MMP-9 in CC cells treated with Dox-Mel combination decreased more significantly, as compared to doxorubicin and melatonin monotherapy. These results showed that doxorubicin and melatonin may inhibit the invasion and migration of CC cells through downregulation of MMPs.

The apoptosis induction is one of the most important mechanisms of cancer treatment with chemotherapeutic drugs. We demonstrated that the apoptosis rate of the CC cells treated with doxorubicin, melatonin, and Dox-Mel were increased, as compared with untreated CC cells. However, the apoptosis rates of CC cells treated with Dox-Mel indicated more significant increase in comparison with doxorubicin and melatonin monotherapies. Previous studies reported that doxorubicin and melatonin increased apoptosis rate in several cancer cells (Di Bella et al., 2013; Wakharde et al., 2018). Several factors have identified in the regulation of apoptotic pathways. In particular, the evidence showed that doxorubicin and melatonin are able to down-regulate and up-regulate the anti-apoptotic and pro-apoptotic factors, respectively (Di Bella et al., 2013; Wakharde et al., 2018). In this regards, we demonstrated that doxorubicin and melatonin were able to down-regulated the mRNA expression of BCL-2 and SURVIVIN genes, as anti-apoptotic factors. Moreover, it was shown doxorubicin and melatonin up-regulated the mRNA expression of $B A X$ and SMAC genes, as pro-apoptotic factors. As expected, combinational treatment with Dox-Mel resulted in the favor of apoptotic pattern of BCL-2, SURVIVIN, BAX, and SMAC genes mRNA expression. Furthermore, we demonstrated that levels of $\mathrm{Bcl}-2$ and Survivin proteins were decreased via doxorubicin and melatonin treatment, which is related to increased viability and proliferation of CC cells, Moreover, the obtained results indicated that doxorubicin and melatonin significantly increased the levels of Bax and Smac proteins, which decreased viability and proliferation of CC cells. However, combinational treatment with Dox-Mel resulted in the favor of apoptotic pattern of Bcl2, Survivin, Bax, and Smac proteins levels. These results showed that doxorubicin and melatonin could inhibit CC cells viability and proliferation through induction of apoptotic pathways. 
The current study represents a novel combination chemotherapeutic approach to treatment of patients with doxorubicin-resistant $\mathrm{CC}$. It was shown that, melatonin enhanced the anticancer activity of doxorubicin through induction of intrinsic apoptotic pathway and suppressed the invasiveness of CC cells. Therefore, the Dox-Mel can be used in the future to control and even treatment of patients with CC. However, further studies are required to identify the enhanced anticancer mechanisms of Dox-Mel to better management of patients with $\mathrm{CC}$ and prescription of lower doses of chemotherapeutic agents.

The current study represents a novel combination chemotherapeutic approach to treatment of patients with doxorubicin-resistant CC. It was shown that, melatonin enhanced the anticancer activity of doxorubicin through induction of intrinsic apoptotic pathway and suppressed the invasiveness of CC cells. Therefore, the Dox-Mel can be used in the future to control and even treatment of patients with CC. However, further studies are required to identify the enhanced anticancer mechanisms of Dox-Mel to better management of patients with $\mathrm{CC}$ and prescription of lower doses of chemotherapeutic agents.

\section{Declarations}

\section{Acknowledgements}

This study was supported by a grant from Immunology Research Center, Tabriz University of Medical Sciences, Tabriz, Iran (Grant No.: 64832, Ethics committee No.: IR.TBZMED.REC.1398.1205).

\section{Author contributions}

Each author has contributed to the design of the study, interpretation of data, manuscript preparation, editing and has provided approval of the final version submitted for publication.

\section{Funding}

Not applicable.

\section{Availability of data and materials}

Not applicable.

\section{Ethics approval and consent to participate}

Not applicable.

\section{Consent for publication}

Not applicable.

\section{Competing interests}

The authors declare that they have no competing interests. 


\section{References}

1. Haggar FA, Boushey RP (2009) Colorectal cancer epidemiology: incidence, mortality, survival, and risk factors. Clin Colon Rectal Surg 22(04):191-7.

2. Wang YJ, Zhang YK, Zhang GN, Al Rihani SB, Wei MN, Gupta P, Zhang XY, Shukla S, Ambudkar SV, Kaddoumi A, Shi Z (2017) Regorafenib overcomes chemotherapeutic multidrug resistance mediated by ABCB1 transporter in colorectal cancer: In vitro and in vivo study. Cancer Lett 396:145-54.

3. Soheilyfar S, Velashjerdi Z, Hajizadeh YS, Maroufi NF, Amini Z, Khorrami A, Azimian SH, Isazadeh A, Taefehshokr S, Taefehshokr N (2018) In vivo and in vitro impact of miR-31 and miR-143 on the suppression of metastasis and invasion in breast cancer. J BUON 23(5):1290-6.

4. Maroufi NF, Vahedian V, Akbarzadeh M, Mohammadian M, Zahedi M, Isazadeh A, Pouremamali F, Taefehshokr S, Heidari M, Rashidi M, Nouri M (2020) The apatinib inhibits breast cancer cell line MDA-MB-231 in vitro by inducing apoptosis, cell cycle arrest, and regulating nuclear factor-KB (NFKB) and mitogen-activated protein kinase (MAPK) signaling pathways. Breast Cancer 1-8.

5. Nakanishi T, Ross DD (2012) Breast cancer resistance protein (BCRP/ABCG2): its role in multidrug resistance and regulation of its gene expression. Chin J Cancer 31(2):73.

6. Hu T, Li Z, Gao CY, Cho CH (2016) Mechanisms of drug resistance in colon cancer and its therapeutic strategies. World J Gastroenterol 22(30):6876.

7. Menéndez-Menéndez J, Hermida-Prado F, Granda-Díaz R, González A, García-Pedrero JM, Del-RíoIbisate N, González-González A, Cos S, Alonso-González C, Martínez-Campa C (2019) Deciphering the molecular basis of melatonin protective effects on breast cells treated with doxorubicin: TWIST1 a transcription factor involved in EMT and metastasis, a novel target of melatonin. Cancers 11(7):1011.

8. Fic M, Podhorska-Okolow M, Dziegiel P, Gebarowska E, Wysocka T, Drag-Zalesinska M, Zabel M (2007) Effect of melatonin on cytotoxicity of doxorubicin toward selected cell lines (human keratinocytes, lung cancer cell lines A-549, laryngeal cancer cell line Hep-2). in vivo 21(3):513-8.

9. Chen X, Hao A, Li X, Du Z, Li H, Wang H, Yang H, Fang Z (2016) Melatonin inhibits tumorigenicity of glioblastoma stem-like cells via the AKT-EZH2-STAT3 signaling axis. J Pineal Res 61(2):208-17.

10. Kim JH, Jeong SJ, Kim B, Yun SM, Choi DY, Kim SH (2012) Melatonin synergistically enhances cisplatin-induced apoptosis via the dephosphorylation of ERK/p90 ribosomal S6 kinase/heat shock protein 27 in SK-OV-3 cells. J Pineal Res 52(2):244-52.

11. Wang J, Hao H, Yao L, Zhang X, Zhao S, Ling EA, Hao A, Li G (2012) Melatonin suppresses migration and invasion via inhibition of oxidative stress pathway in glioma cells. J Pineal Res 53(2):180-7.

12. Maroufi NF, Vahedian V, Hemati S, Rashidi M, Akbarzadeh M, Zahedi M, Pouremamali F, Isazadeh A, Taefehshokr S, Hajazimian S, Seraji N (2020) Targeting Cancer Stem Cells by Melatonin: Effective Therapy for Cancer Treatment. Pathol Res Pract 152919.

13. Witt-Enderby PA, Radio NM, Doctor JS, Davis VL (2006) Therapeutic treatments potentially mediated by melatonin receptors: potential clinical uses in the prevention of osteoporosis, cancer and as an 
adjuvant therapy. J Pineal Res 41(4):297-305.

14. Kong PJ, Byun JS, Lim SY, Lee JJ, Hong SJ, Kwon KJ, Kim SS (2008) Melatonin induces Akt phosphorylation through melatonin receptor-and PI3K-dependent pathways in primary astrocytes. Korean J Physiol Pharmacol 12(2):37-41.

15. Mao L, Yuan L, Slakey LM, Jones FE, Burow ME, Hill SM (2010) Inhibition of breast cancer cell invasion by melatonin is mediated through regulation of the p38 mitogen-activated protein kinase signaling pathway. Breast Cancer Res 12(6):R107.

16. Menéndez-Menéndez J, Martínez-Campa C (2018) Melatonin: an anti-tumor agent in hormonedependent cancers. Int J Endocrinol 2018.

17. Najafi M, Salehi E, Farhood B, Nashtaei MS, Hashemi Goradel N, Khanlarkhani N, Namjoo Z, Mortezaee K (2019) Adjuvant chemotherapy with melatonin for targeting human cancers: A review. J Cell Physiol 234(3):2356-72.

18. Vahedian V, Asadi A, Esmaeili P, Zamani S, Zamani R, Hajazimian S, Isazadeh A, Shanehbandi D, Maroufi NF (2020) Anti-inflammatory activity of emu oil-based nanofibrous scaffold through downregulation of IL-1, IL-6, and TNF-a pro-inflammatory cytokines. Horm Mol Biol Clin Investig 20190052.

19. Firouzi Amoodizaj F, Baghaeifar S, Taheri E, Farhoudi M, Safi M, Seyyed Sani N, Hajazimian S, Isazadeh A, Shanehbandi D (2020) Enhanced Anticancer Potency of Doxorubicin in Combination with Curcumin in Gastric Adenocarcinoma. J Biochem Mol Toxicol e22486.

20. Hajazimian S, Maleki M, Danaei Mehrabad S, Isazadeh A (2020) Human Wharton's Jelly Stem Cells Inhibit Endometriosis through Apoptosis Induction. Reproduction 159(7):549-558.

21. Mahdavi S, Kheyrollahi M, Sheikhloei H, Isazadeh A (2019) Antibacterial and Antioxidant Activities of Essential Oil on Food Borne Bacteria. Open Microbiol J 13:81-85.

22. Xu Z, Chen L, Xiao Z, Zhu Y, Jiang H, Jin Y, Gu C, Wu Y, Wang L, Zhang W, Zuo J (2018) Potentiation of the anticancer effect of doxorubicinin drug-resistant gastric cancer cells by tanshinone IIA. Phytomedicine 51:58-67.

23. Zhang D, Fan D (2010) New insights into the mechanisms of gastric cancer multidrug resistance and future perspectives. Future Oncol 6(4):527-37.

24. Hosseinzadeh L, Behravan J, Mosaffa F, Bahrami G, Bahrami AR, Karimi G (2011) Effect of curcumin on doxorubicin-induced cytotoxicity in H9c2 cardiomyoblast cells. Iran J Basic Med Sci 14(1):49-56.

25. Bakhshaiesh TO, Armat M, Shanehbandi D, Sharifi S, Baradaran B, Hejazi MS, Samadi N (2015) Arsenic trioxide promotes paclitaxel cytotoxicity in resistant breast cancer cells. Asian Pac J Cancer Prev 16(13):5191-7.

26. Di Bella G, Mascia F, Gualano L, Di Bella L (2013) Melatonin anticancer effects. Int J Mol Sci 14(2):2410-30.

27. Wakharde AA, Awad AH, Bhagat A, Karuppayil SM (2018) Synergistic Activation of Doxorubicin against Cancer: A Review. Am J Clin Microbiol Antimicrob 1(2):1009. 
Figures
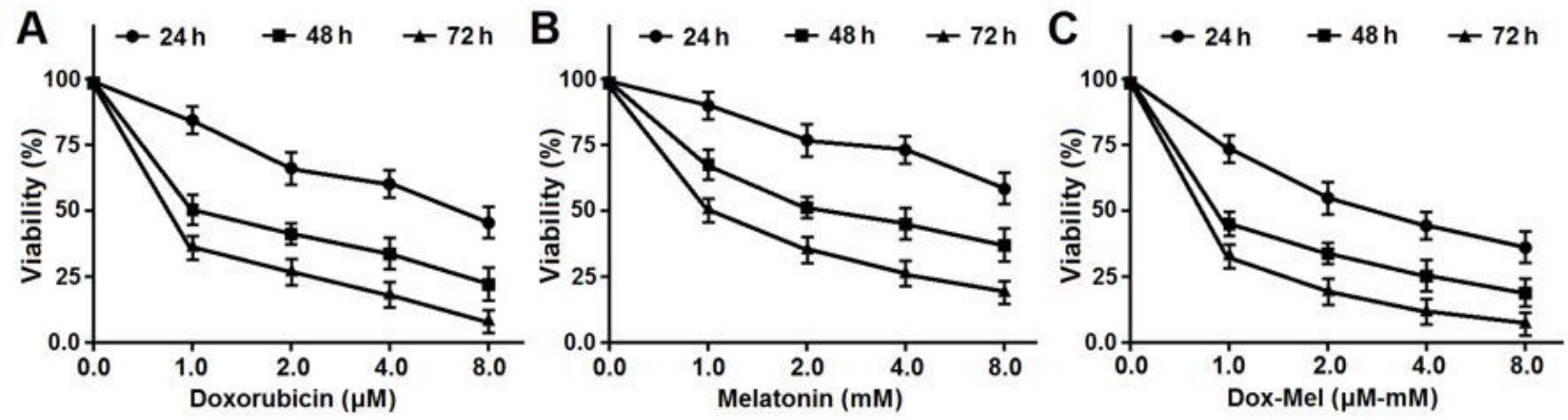

Figure 1

The inhibitory effects of doxorubicin, melatonin, and Dox-Mel on viability of CC cells in 24 (A), 48 (B), and 72 (C) hours. The anticancer activity of Dox-Mel was significantly more than doxorubicin and melatonin alone.

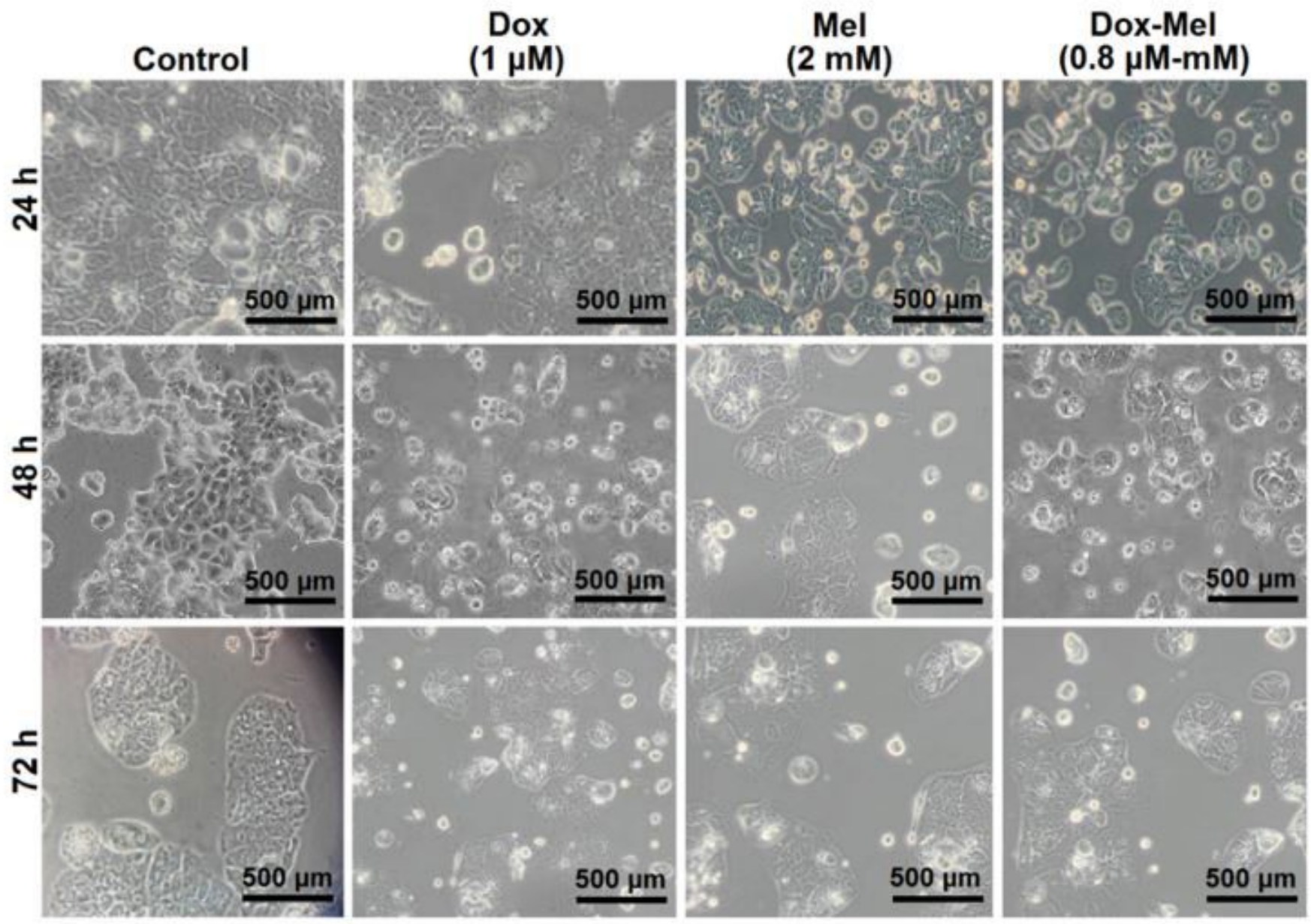

Figure 2 
The morphological alteration of CC cells treated with doxorubicin $(1 \mu \mathrm{M})$, melatonin $(2 \mathrm{mM})$, and Dox-Mel $(0.8 \mu \mathrm{M}-\mathrm{mM})$ in 24,48 , and 72 hours. Treatment with Dox-Mel caused more profound morphological alterations.
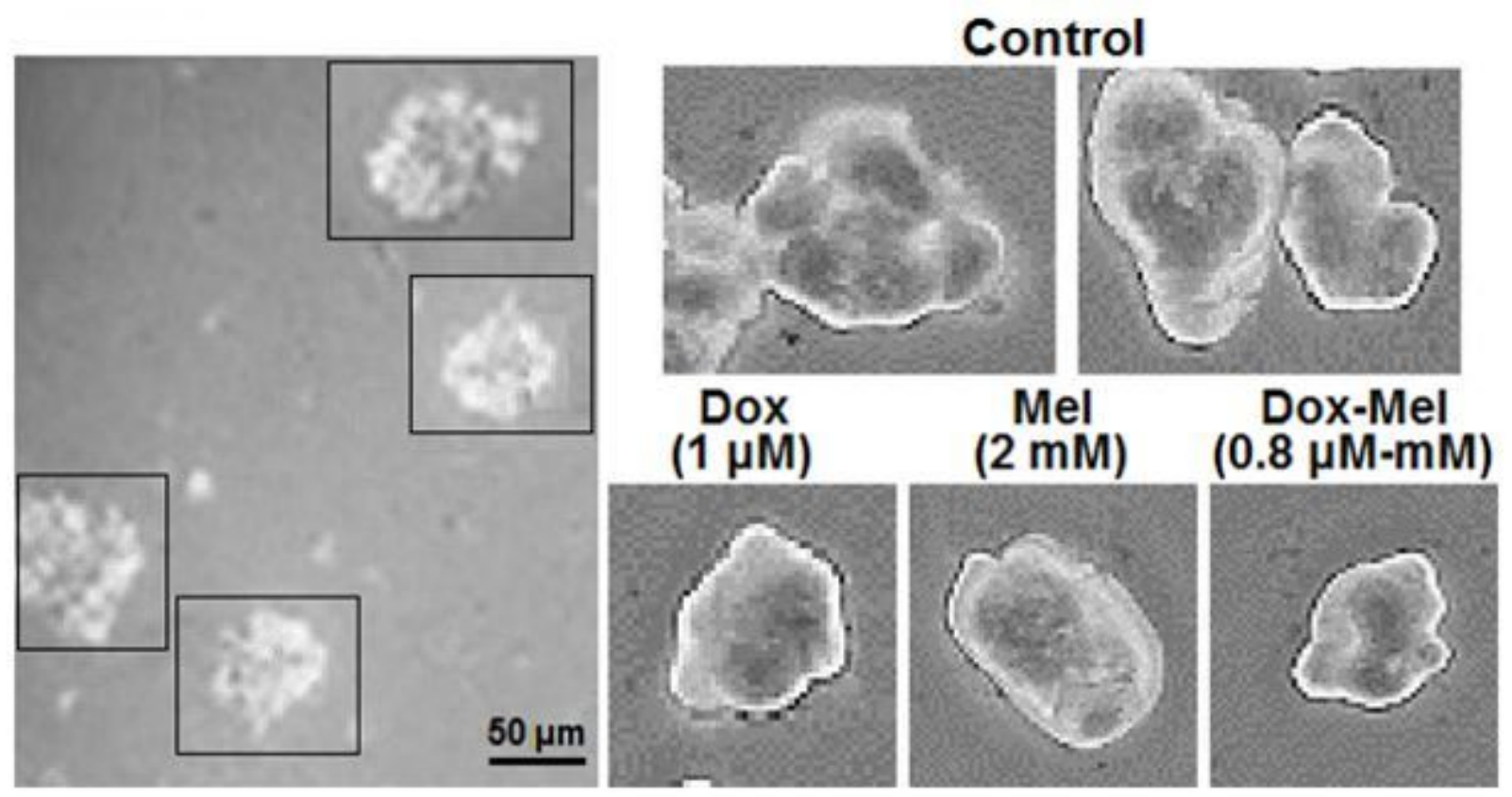

\section{Figure 3}

The tumor spheroid formation of CC cells treated with doxorubicin $(1 \mu \mathrm{M})$, melatonin $(2 \mathrm{mM})$, and DoxMel $(0.8 \mu \mathrm{M}-\mathrm{mM})$ after 7-12 days. Treatment of CC cells with Dox-Mel cause significant decrease in the size and number of tumor spheroids. 

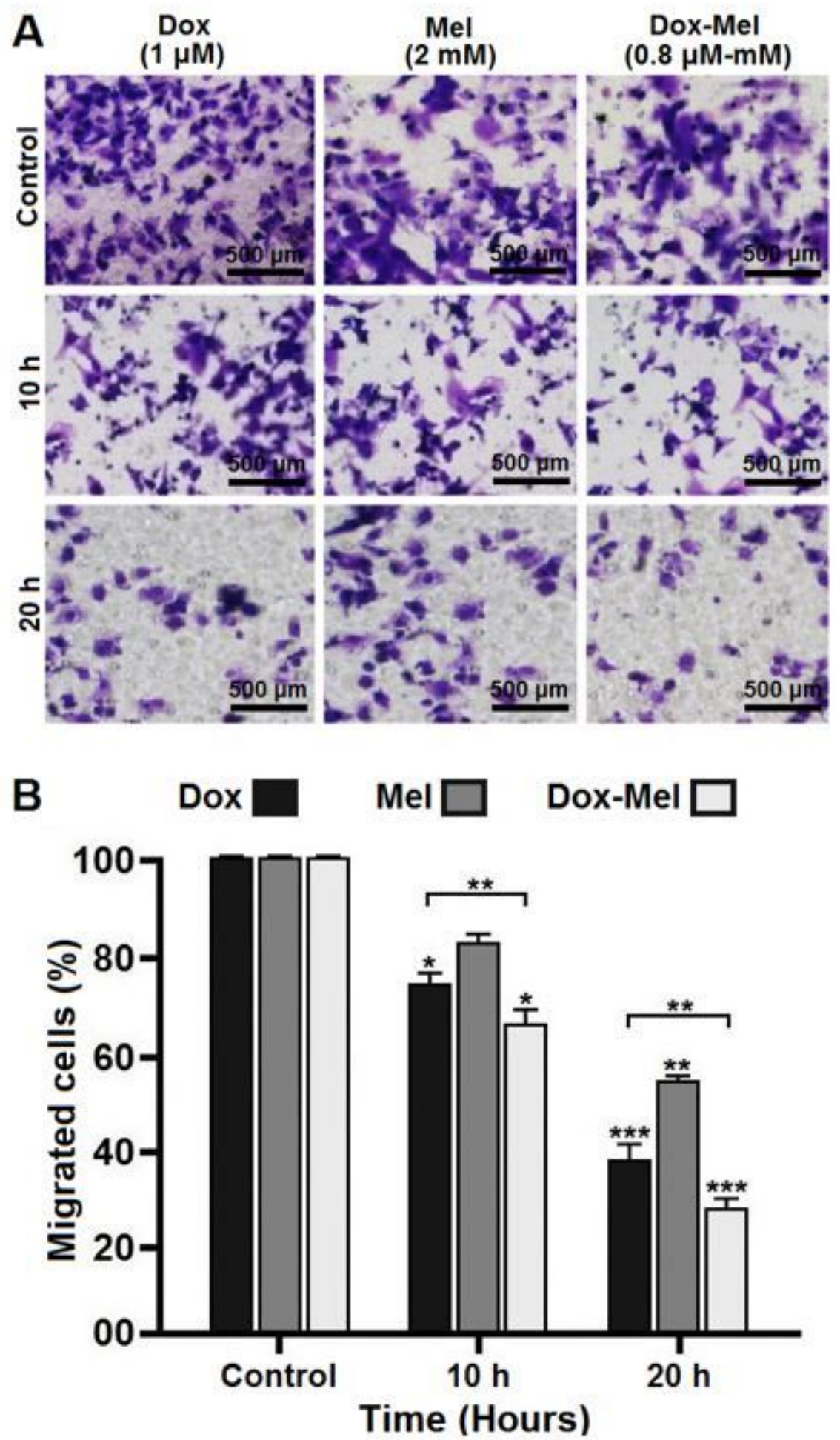

Figure 4

The migration and invasion of CC cells evaluated by trans-well migration assay following treatment with doxorubicin $(1 \mu \mathrm{M})$, melatonin $(2 \mathrm{mM})$, and Dox-Mel $(0.8 \mu \mathrm{M}-\mathrm{mM})$ for $10-20$ hours $(\mathrm{A})$. Treatment of CC cells with Dox-Mel caused more significant decrease of migration and invasion (B). 


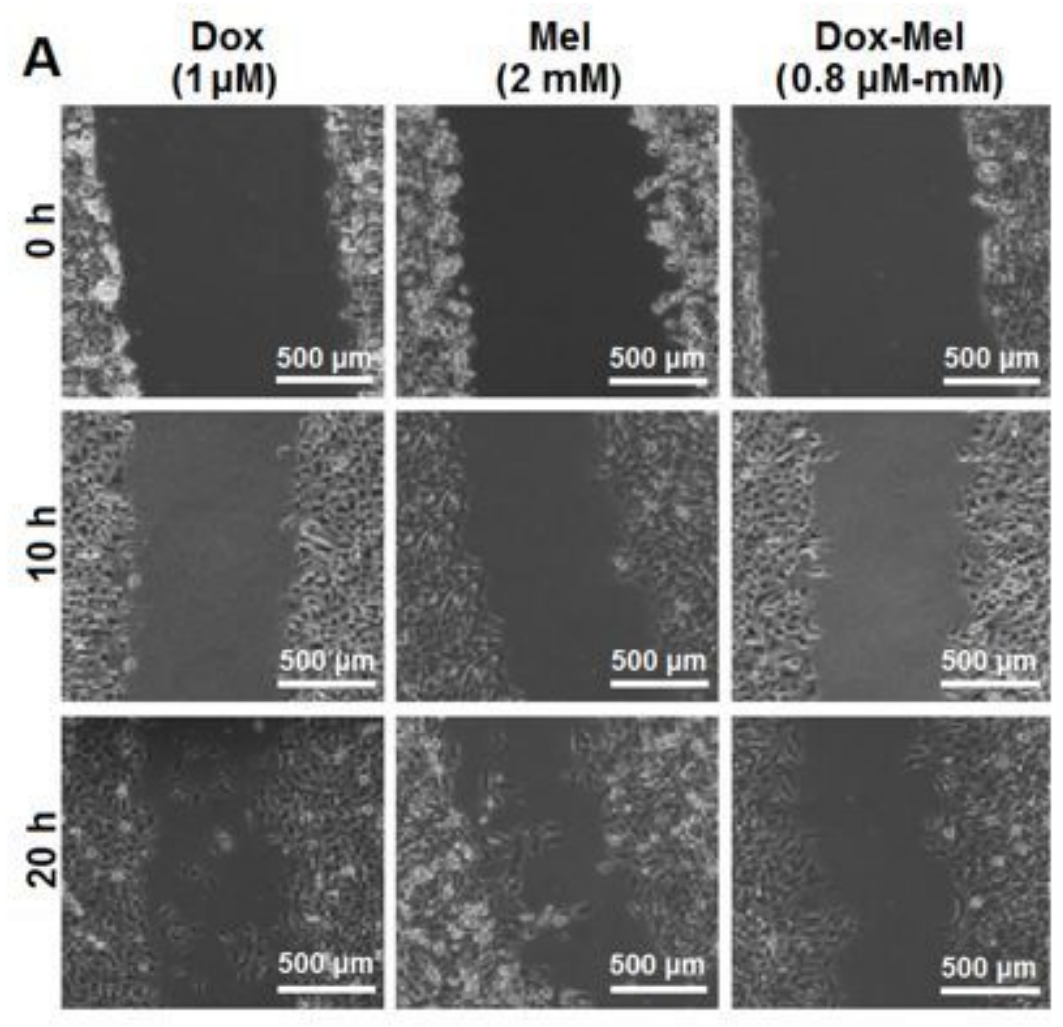

B Dox $\square \quad$ Mel $\square$ Dox-Mel $\square$

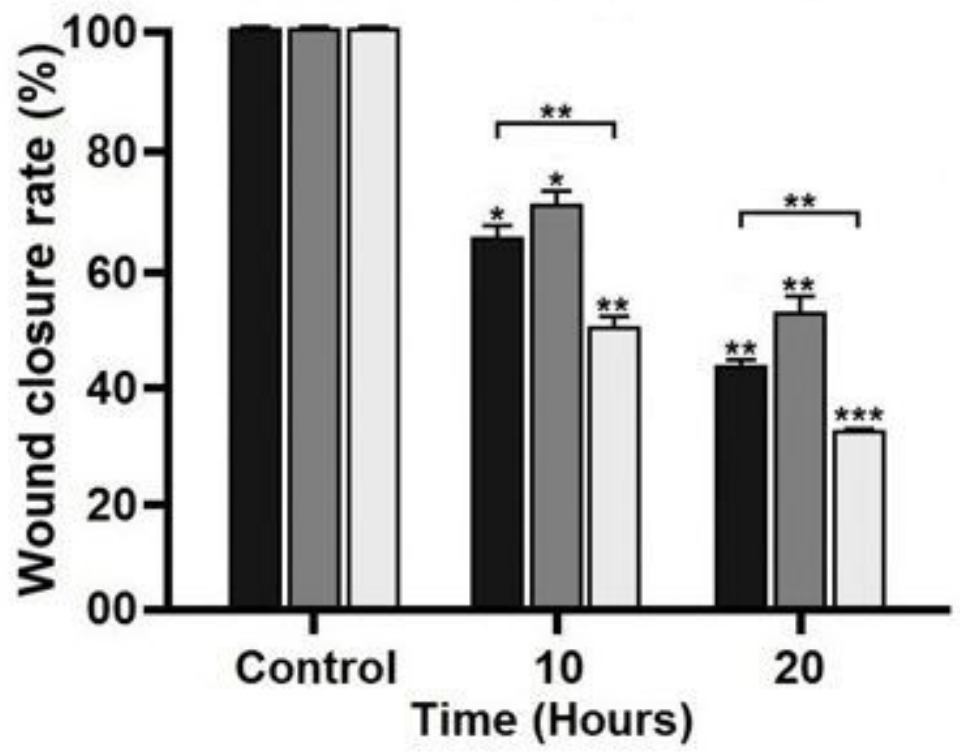

Figure 5

The migration and invasion of $\mathrm{CC}$ cells evaluated by scratch wound healing assay following treatment with doxorubicin $(1 \mu \mathrm{M})$, melatonin $(2 \mathrm{mM})$, and Dox-Mel $(0.8 \mu \mathrm{M}-\mathrm{mM})$ for 10-20 hours $(\mathrm{A})$. Treatment of $\mathrm{CC}$ cells with Dox-Mel resulted in more significant decrease of migration and invasion (B). 

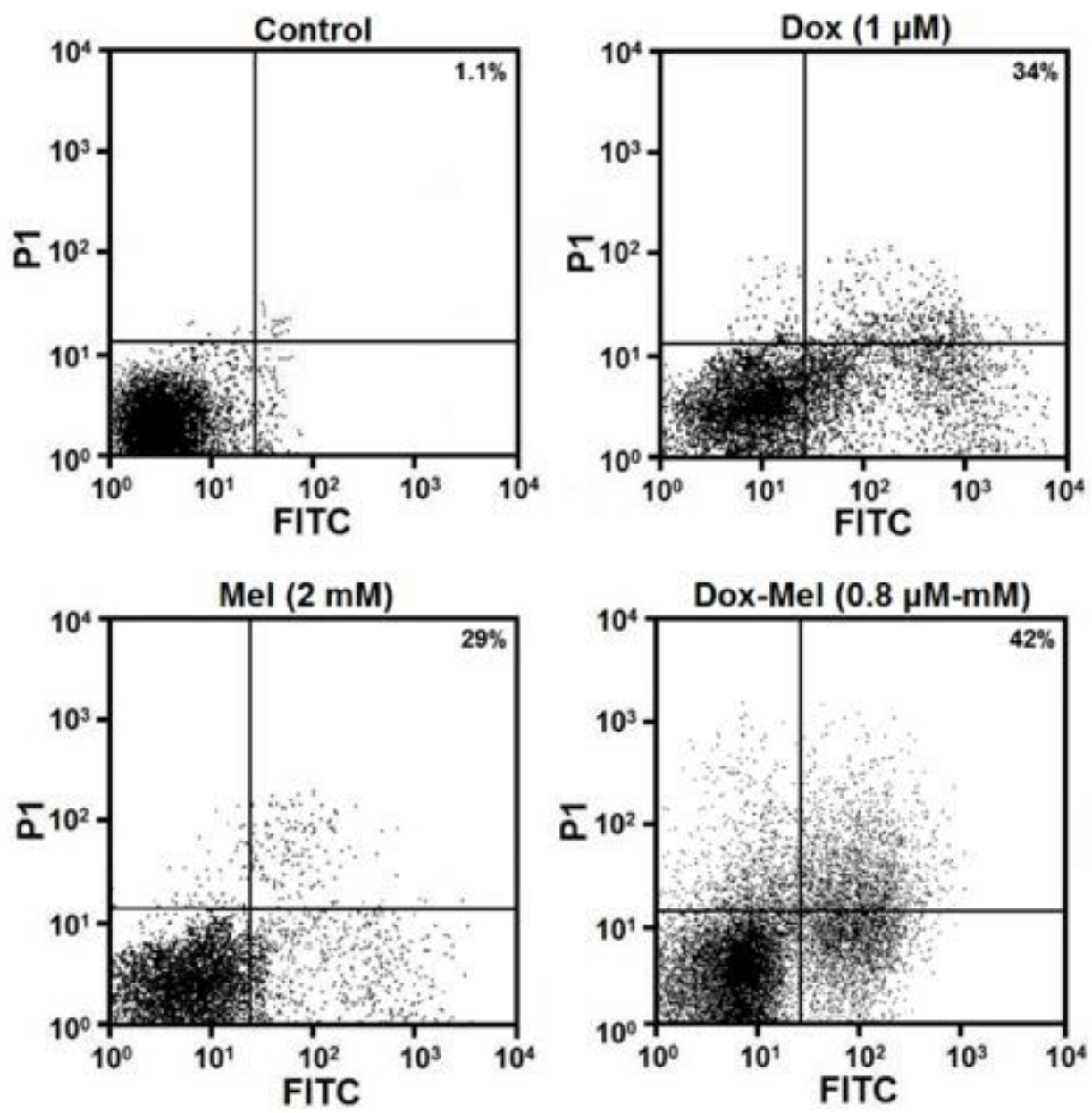

Figure 6

The apoptosis rate of CC cells treated with doxorubicin $(1 \mu \mathrm{M})$, melatonin $(2 \mathrm{mM})$, and Dox-Mel $(0.8 \mu \mathrm{M}-$ $\mathrm{mM})$. The induction of apoptosis in the CC cells treated with Dox-Mel was significantly more than doxorubicin and melatonin alone. 

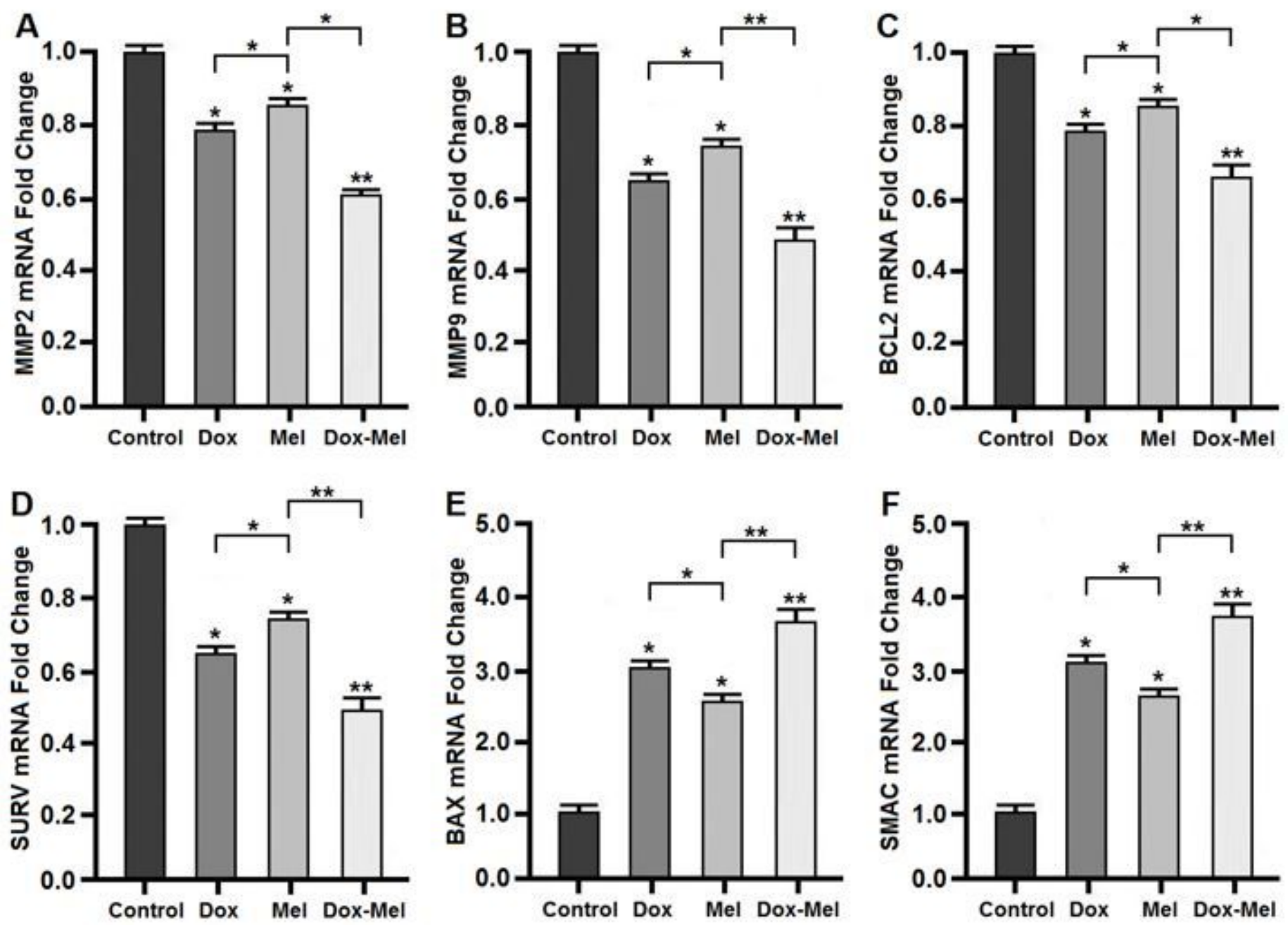

Figure 7

The mRNA expression analysis of migration and invasion related MMP-2 (A) and MMP-9 genes (B) and apoptosis related BCL-2 (C), SURVIVIN (D), BAX (E), and SMAC (F) genes by qRT-PCR following the treatment with doxorubicin $(1 \mu \mathrm{M})$, melatonin $(2 \mathrm{mM})$, and Dox-Mel $(0.8 \mu \mathrm{M}-\mathrm{mM})$. The regulation of migration and invasion and apoptosis related genes expression in the CC cells treated with Dox-Mel was significantly more than doxorubicin and melatonin alone. 

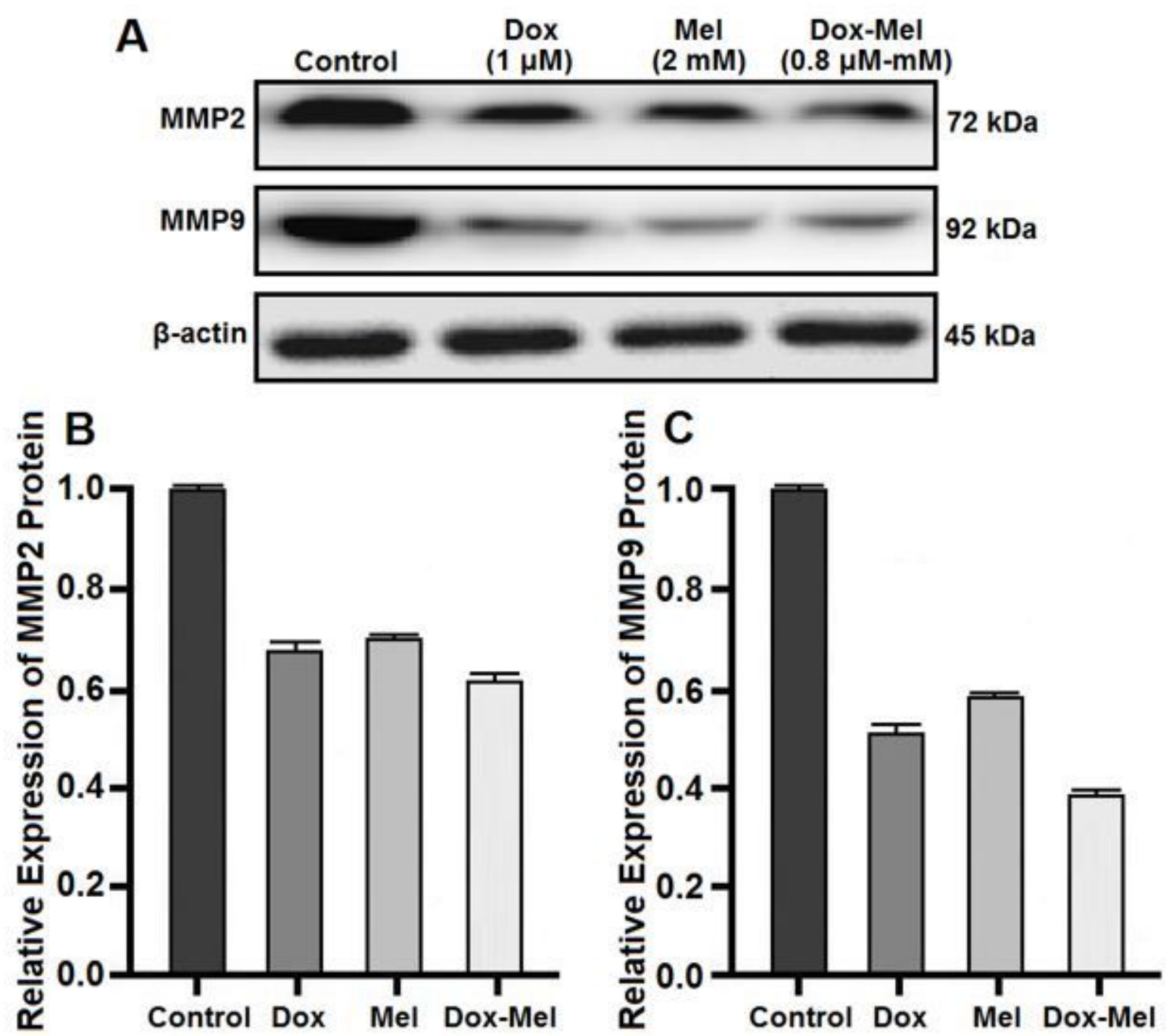

Figure 8

The western blot analysis (A) of MMP-2 (B) and MMP-9 (C) proteins following the treatment with doxorubicin $(1 \mu \mathrm{M})$, melatonin $(2 \mathrm{mM})$, and Dox-Mel $(0.8 \mu \mathrm{M}-\mathrm{mM})$. The regulation of migration and invasion related genes expression in the CC cells treated with Dox-Mel was significantly more than doxorubicin and melatonin alone. 
A
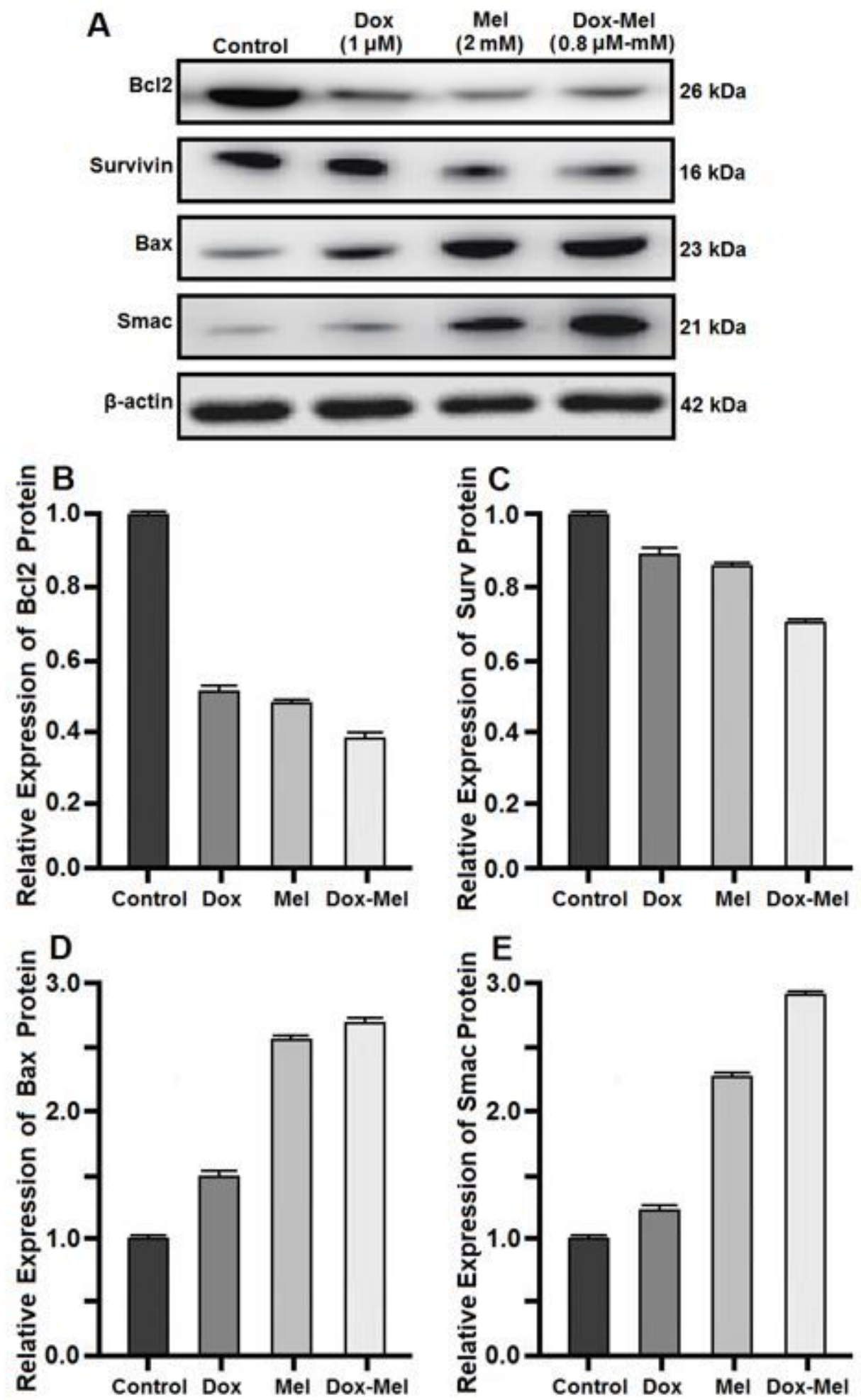

\section{Figure 9}

The western blot analysis (A) of Bcl-2 (B), Survivin (C), Bax (D), and Smac (E) proteins levels following the treatment with doxorubicin $(1 \mu \mathrm{M})$, melatonin $(2 \mathrm{mM})$, and Dox-Mel $(0.8 \mu \mathrm{M}-\mathrm{mM})$. The regulation of apoptosis related genes expression in the CC cells treated with Dox-Mel was significantly more than doxorubicin and melatonin alone. 